\title{
Evaluation of Candidate Reference Genes for Normalization of Quantitative RT-PCR in Soybean Tissues under Various Abiotic Stress Conditions
}

\author{
Dung Tien Le', Donavan L. Aldrich ${ }^{29}$, Babu Valliyodan ${ }^{29}$, Yasuko Watanabe ${ }^{1}$, Chien Van Ha ${ }^{1}$,

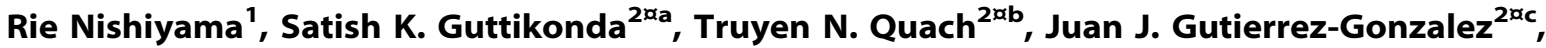 \\ Lam-Son Phan Tran ${ }^{1 *}$, Henry T. Nguyen ${ }^{2 *}$
}

1 Signaling Pathway Research Unit, RIKEN Plant Science Center, Yokohama, Kanagawa, Japan, 2 National Center for Soybean Biotechnology and Division of Plant Sciences, University of Missouri, Columbia, Missouri, United States of America

\begin{abstract}
Quantitative RT-PCR can be a very sensitive and powerful technique for measuring differential gene expression. Changes in gene expression induced by abiotic stresses are complex and multifaceted, which make determining stably expressed genes for data normalization difficult. To identify the most suitable reference genes for abiotic stress studies in soybean, 13 candidate genes collected from literature were evaluated for stability of expression under dehydration, high salinity, cold and ABA (abscisic acid) treatments using delta CT and geNorm approaches. Validation of reference genes indicated that the best reference genes are tissue- and stress-dependent. With respect to dehydration treatment, the Fbox/ABC, Fbox/60s gene pairs were found to have the highest expression stability in the root and shoot tissues of soybean seedlings, respectively. Fbox and 60s genes are the most suitable reference genes across dehydrated root and shoot tissues. Under salt stress the ELF1b/IDE and Fbox/ELF1b are the most stably expressed gene pairs in roots and shoots, respectively, while 60s/Fbox is the best gene pair in both tissues. For studying cold stress in roots or shoots, IDE/60s and Fbox/Act27 are good reference gene pairs, respectively. With regard to gene expression analysis under ABA treatment in either roots, shoots or across these tissues, 60s/ELF1b, ELF1b/Fbox and 60s/ELF1b are the most suitable reference genes, respectively. The expression of ELF1b/ 60 s, 60s/Fbox and 60s/Fbox genes was most stable in roots, shoots and both tissues, respectively, under various stresses studied. Among the genes tested, 60 s was found to be the best reference gene in different tissues and under various stress conditions. The highly ranked reference genes identified from this study were proved to be capable of detecting subtle differences in expression rates that otherwise would be missed if a less stable reference gene was used.
\end{abstract}

Citation: Le DT, Aldrich DL, Valliyodan B, Watanabe Y, Ha CV, et al. (2012) Evaluation of Candidate Reference Genes for Normalization of Quantitative RT-PCR in Soybean Tissues under Various Abiotic Stress Conditions. PLoS ONE 7(9): e46487. doi:10.1371/journal.pone.0046487

Editor: Christian Schönbach, Kyushu Institute of Technology, Japan

Received January 27, 2012; Accepted September 2, 2012; Published September 28, 2012

Copyright: (C) 2012 Le et al. This is an open-access article distributed under the terms of the Creative Commons Attribution License, which permits unrestricted use, distribution, and reproduction in any medium, provided the original author and source are credited.

Funding: This project was supported by the National Center for Soybean Biotechnology and the Missouri Soybean Merchandising Council. The funders had no role in study design, data collection and analysis, decision to publish, or preparation of the manuscript.

Competing Interests: The authors have declared that no competing interests exist.

*E-mail: tran@psc.riken.jp (L-SPT); mailto:nguyenhenry@missouri.edu (HTN)

9 These authors contributed equally to this work.

aa Current address: Biotechnology Regulatory Sciences, Regulatory Sciences and Government Affairs, Dow AgroSciences, Indianapolis, Indiana, United States of America

ab Current address: The Center for Plant Science Innovation, University of Nebraska, Lincoln, Nebraska, United States of America

ac Current address: USDA-ARS Plant Science Research Unit and Department of Agronomy and Plant Genetics, University of Minnesota, St Paul, Minnesota, United States of America

\section{Introduction}

The study of plant adaptive responses to abiotic stresses, such as drought, high salinity and cold stress, is a rapidly growing field of research due largely to its immense impact on global food supply. Abiotic stressors are the adverse environmental conditions which are unfavorable to plant growth and include such circumstances as flooding, extreme temperatures, high soil salinity, and drought. These stressors can have detrimental effects on plants which generally result in major yield losses for the economically important crops, including soybean [1-5]. Understanding the mechanisms plants use to cope with such stresses is crucial for areas of research aimed at the engineering of soybean cultivars with increased stress tolerance [6-9]. Of the various environmen- tal stresses, drought is one which typically receives much attention due to its pervasiveness as well as the significance of its impact to soybean yields worldwide $[3,10]$.

Plants have always evolved under the highly selective pressure of these stresses and have thus established exceedingly complex and broad-stroked genetic and molecular mechanisms to survive the adverse conditions imposed by them. It has been demonstrated in many instances that many of the primary mechanisms plants use to cope with abiotic stress are not constitutively active throughout their lifetime, but are induced at a transcriptional level when these stresses are present [6,9,11]. Therefore, much of the research related to abiotic stress is focused on determining the key factors involved in such responses, and elucidating the network of genes 
induced and/or regulated by these factors is one of the most powerful approaches researchers can employ to study stress tolerance $[9,12-18]$. Consequently, the ability to perform highthroughput profiling sensitive enough to detect subtle changes in the expression levels of a magnitude of target genes is a valuable tool for research focused on abiotic stress-induced gene expression $[1,10,19]$.

Three profiling techniques are currently available to study the changes in gene expression induced by abiotic stress factors. Northern blot is the first technique to have emerged which offered the capability to measure differential gene expression. This technique typically involves using electrophoretically separated RNA which is then transferred to a membrane and hybridized with a detectable complementary probe [20]. But northern blots are both time consuming and labor intensive. And although the sensitivity threshold is quite high, the qualitative nature of the technique limits the ability to accurately quantify expression levels. This, in conjunction with the low throughput aspect of northern blotting, restricts its effectiveness for expression profiling the magnitude of target genes often required for abiotic stress studies. The emergence of DNA microarray hybridization, using either cDNA microarrays or oligonucleotide microarrays [21-23], has enabled the ability to effectively screen a large number of target genes under various conditions, including environmental stimuli [24-29]. This technology uses an arrayed series of microscopic spots of DNA sequences representing individual target genes [30]. For expression profiling studies using two-color microarray, template cDNAs from control and treated samples labeled with different fluorophores are hybridized to the array, and the fluorescent signals from both fluorophores are determined for each probe spot. The differences between signal intensities from each fluorophore are used to determine the changes in gene expression between the control and treatment samples [31]. On the other hand, one-color microarrays provide intensity data for each probe which together indicate a relative level of hybridization with the labeled target [32]. The newly emerging whole-genome tiling arrays covering whole genome sequence of both strands with oligo probes have allowed us to identify many stress-inducible genes and transcripts including non-protein-coding RNAs, which were not able to be unidentified by the use of cDNA microarrays and oligonucleotide microarrays [33,34]. Although all of these microarray platforms enable us to screen thousands of gene probes simultaneously, microarray technology is not sensitive enough for detecting modest changes in gene expression [35]. Additionally, the specificities of the probe oligonucliotides in these arrays are not always high enough to ensure that individual genes are being hybridized.

Quantitative real-time polymerase chain reaction (qRT-PCR) is potentially the most sensitive method developed for detecting the changes in gene expression [36]. This process uses fluorescent dyes or probes to detect the amount of double-stranded DNA amplified from template DNA during a series of sequential PCR reactions using forward and reverse primer sequences designed to amplify a segment of a particular target gene [37,38]. The fractional PCR cycle number at which this fluorescent signal achieves a defined threshold is referred to as the Ct value and is proportional to the abundance of that target gene in the template DNA. Like DNA microarray hybridization, qRT-PCR has the ability to perform screening of a multitude of gene targets. However, unlike DNA microarrays, the nature of the PCR reaction intrinsic to this method allows for both a high sensitivity of detection as well as the use of high specificity primers to ensure that the expression levels of individual genes are being measured. These aspects of qRTPCR make it an attractive tool for use in research related to studying the changes in gene expressions induced by abiotic stresses in plants [23,39-42].

However, as powerful and sensitive as qRT-PCR has the potential to be, it is not without its pitfalls. A fundamental constraint on the accurate interpretation of qRT-PCR data is the necessity of a stable, constitutively-expressed reference gene which can be used as a standard measure for comparing one sample to another [43]. This normalization is typically performed in order to compensate for the variability between samples contributed by the multitude of factors which can influence the cycle threshold (CT) value obtained from a qRT-PCR reaction. Ideal reference genes for the normalization of qRT-PGR data are genes which demonstrate a consistent level of expression among control and treated samples, and in the context of studies under stresses, the expression levels of these reference genes would be unaffected by the stress treatments applied and would allow for the accurate normalization of target gene expression levels between samples. The changes of gene expression levels induced by abiotic stresses like drought, high salinity and cold can be complex and multifaceted, often affecting the expression levels of genes which would otherwise be stable and suitable as reference genes in other experimental circumstances. Consequently, it is crucial that the expression stability of potential reference gene be confirmed under abiotic stress conditions before they are utilized for the normalization of qRT-PCR data generated in these studies. For soybean, several reference genes have been available; however, these genes were validated only under normal or biotic stress conditions [44 46]. An extended search in literature identified an article on the journal of Pesquisa Agropecuária Brasileira by Stolf-Moreira and coworkers which described the search for reference genes for use in qRT-PCR analysis of soybean under drought condition only [47]. Therefore, the primary objective of this study was to determine in systematic manner reference genes which demonstrate a high degree of expression stability under various abiotic stress conditions in soybean, to facilitate a more accurate normalization of qRT-PGR assays.

\section{Materials and Methods}

\section{Plant materials, growth conditions and treatments}

Soybean cv. Williams 82 seeds were germinated in 6-litre pots containing vermiculite soil and grown under greenhouse conditions (continuous $30^{\circ} \mathrm{C}$ temperature, photoperiod of $12 \mathrm{~h} / 12 \mathrm{~h}$, $80 \mu \mathrm{mol} \mathrm{m}-2 \mathrm{~s}-1$ photon flux density and $40-60 \%$ relative humidity). For non-stress treatment all the plants were watered at regular intervals. For stress treatment, 12-d-old plants were carefully removed from soil, and roots were gently washed to remove soil. Dehydration treatment was performed as described in [40]. For NaCl and ABA (abscisic acid) treatments, 12-d-old seedlings were immersed in a solution containing either $200 \mathrm{mM}$ $\mathrm{NaCl}$ and $100 \mu \mathrm{M} \mathrm{ABA}$, respectively, for 0,2 , and $10 \mathrm{~h}$. Cold treatment was performed by transferring 12-d-old plants to a container maintained at $4^{\circ} \mathrm{C}$ for 0,2 and $10 \mathrm{~h}$. A set of plant samples was also maintained in water at room temperature for the same durations as control. After the treatments, root and shoot samples were separately collected in three biological repeats for expression analyses.

\section{RNA isolation, DNAse treatment, and cDNA synthesis}

Total RNA was isolated as described by using TRIZOL reagent (Invitrogen) according to the protocol provided by the manufacturer. RNA concentration and integrity were measured prior to DNase digestion with the NanoDrop UV-Vis spectrophotometer (NanoDrop Technologies). For each sample, $4 \mu \mathrm{g}$ of total RNA was digested in a volume of $25 \mu \mathrm{l}$ with Turbo DNA-free DNase I (Ambion). First-strand cDNA synthesis was performed using $1 \mu \mathrm{g}$ 


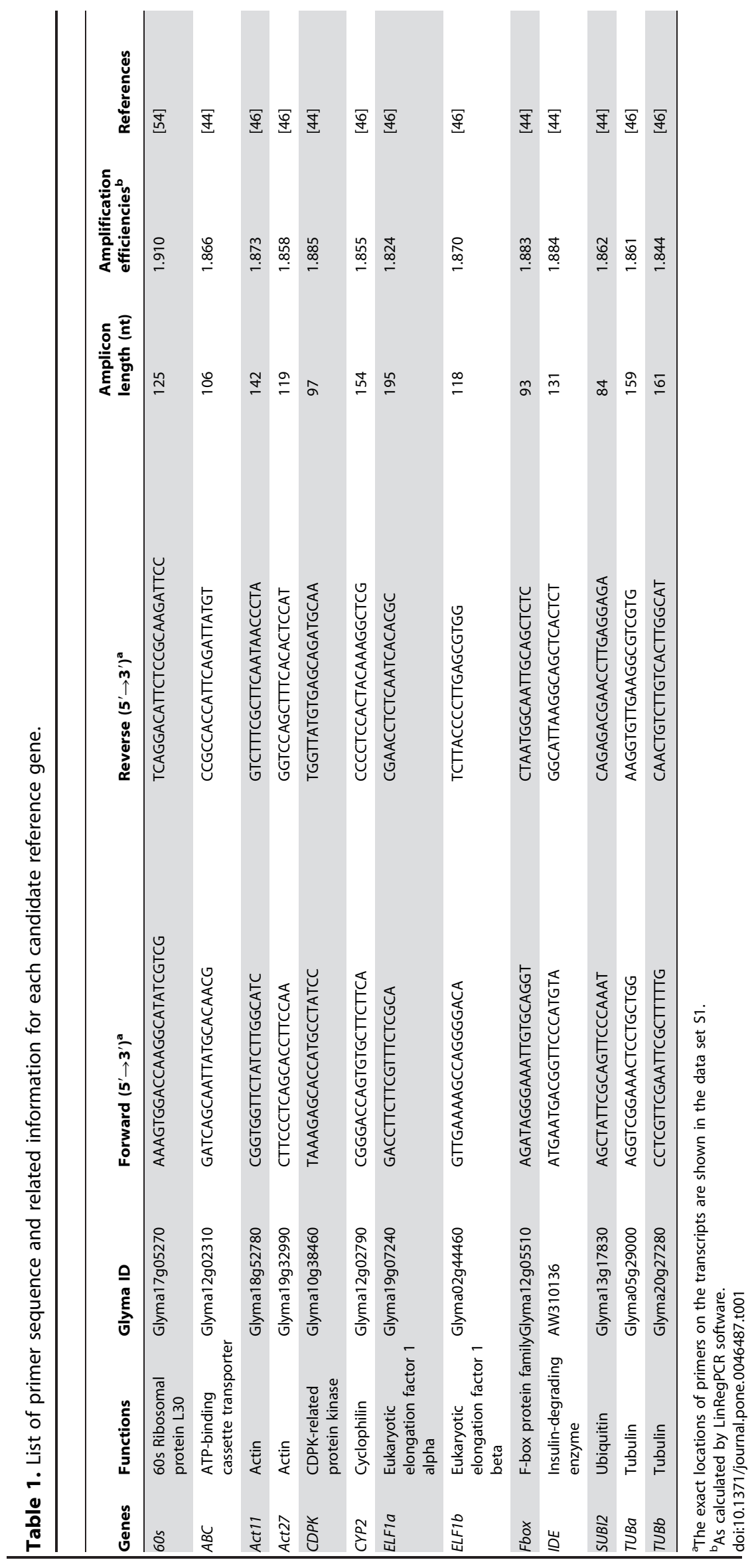


Table 2. Cycle thresholds and average of standard deviations (STDEVP) of delta CT obtained from tissues under all stress combinations.

\begin{tabular}{|c|c|c|c|c|c|c|c|}
\hline & \multicolumn{2}{|c|}{ Mean CT } & \multicolumn{2}{|c|}{ STDEVP of CT } & \multicolumn{3}{|c|}{ Average of STDEVP of $\Delta C T$} \\
\hline & Roots & Shoots & Roots & Shoots & Roots & Shoots & $\begin{array}{l}\text { Roots \& } \\
\text { Shoots }\end{array}$ \\
\hline $60 \mathrm{~s}$ & 21.31 & 21.97 & 0.5507 & 0.8184 & 0.4274 & 0.5112 & 0.4492 \\
\hline$A B C$ & 23.64 & 24.38 & 0.5522 & 0.6079 & 0.4580 & 0.5851 & 0.5238 \\
\hline Act27 & 21.64 & 22.03 & 0.5793 & 1.0963 & 0.5325 & 0.6736 & 0.5837 \\
\hline Act11 & 19.11 & 19.70 & 0.7787 & 0.7892 & 0.5772 & 0.5761 & 0.5682 \\
\hline$C D P K$ & 25.79 & 26.35 & 0.8712 & 0.8752 & 0.7810 & 0.8356 & 0.8015 \\
\hline CYP2 & 17.63 & 17.68 & 0.5748 & 0.5359 & 0.5148 & 0.5897 & 0.5987 \\
\hline ELF1a & 18.41 & 18.87 & 0.5853 & 0.7598 & 0.5210 & 0.5905 & 0.5541 \\
\hline$E L F 1 b$ & 20.67 & 21.86 & 0.5545 & 0.7702 & 0.4204 & 0.5462 & 0.5233 \\
\hline Fbox & 21.09 & 21.55 & 0.5217 & 0.6606 & 0.4908 & 0.5115 & 0.4843 \\
\hline$I D E$ & 21.66 & 22.41 & 0.7632 & 0.6132 & 0.5284 & 0.5518 & 0.5333 \\
\hline$S U B I 2$ & 25.30 & 26.65 & 1.1050 & 0.8995 & 0.9455 & 1.1124 & 1.0802 \\
\hline$T U B a$ & 20.12 & 20.34 & 0.6796 & 1.0552 & 0.8019 & 1.1125 & 0.9594 \\
\hline$T U B b$ & 20.60 & 21.16 & 0.6805 & 1.0289 & 0.5258 & 0.7495 & 0.6293 \\
\hline
\end{tabular}

Data obtained for the top five genes are shown in bold letters, while those for the top two genes are in italic and bold letters

doi:10.1371/journal.pone.0046487.t002

of DNase I-treated RNA with the ReverTra Ace ${ }^{\circledR}$ qPCR RT Kit (Toyobo, Japan) in a 20- $\mu$ l reaction volume according to the manufacturer's supplied protocol. All procedures were performed essentially as previously reported [40].

\section{qRT-PCR and data analyses}

qRT-PCRs were performed in 96-well plates on a Stratagene MX3000P system (Agilent Technologies, Santa Clara, CA, USA) using Thunderbird ${ }^{\mathrm{TM}}$ SYBR $^{\circledR}$ qPGR Mix (Toyobo, Japan). Primer sets $(0.4 \mu \mathrm{M}$ final concentrations for each primer) were used in a final volume of $10 \mu \mathrm{l}$ per well. The thermal profile of the qRT-PCRs was at $95^{\circ} \mathrm{C}$ for $1 \mathrm{~min}, 40$ cycles at $95^{\circ} \mathrm{C}$ for $15 \mathrm{~s}$ and at $60^{\circ} \mathrm{C}$ for $1 \mathrm{~min}$. Dissociation curves (Figure $\mathrm{S} 1$ ) were obtained using a thermal melting profile performed after the last PCR cycle: $95^{\circ} \mathrm{C}$ for $15 \mathrm{~s}$ followed by a constant increase in the temperature between $60^{\circ} \mathrm{C}$ and $95^{\circ} \mathrm{C}$. Background-corrected raw fluorescence data were exported from the MX3000P system and analyzed in LinRegPCR software with a built-in baseline correction and amplification efficiency calculation [48,49]. Amplicon-based fluorescence thresholds were used to obtain the CT values. For confirmation of primer specificity, amplicon length was verified by electrophoresis of products through a $2 \%$ agarose gel (data not shown). Delta CT analyses were performed essentially as described by Silver et al. [50]. The mean of standard deviations of delta CTs was used to rank the performance of each candidate reference gene. The lower the values are, the more stable the expression of the candidate genes is (Table S1).

\section{Results and Discussion}

Screening of universal candidate reference genes for dehydration, high-salinity, cold and ABA treatments

The strategy conceived for determining effective reference genes in soybean began with a screening of the candidate genes whose expression were commonly stable across the major stressors, including dehydration, salt and cold stresses, which are often encountered by soybean plants $[1,3]$. Gene expression in response to abiotic stress has been known to be regulated in ABAdependent and/or ABA-independent manner [39,51-53]; thus, we also included ABA treatment into our study. A qRT-PCR assay was designed to measure the expression stability between control and stress- or ABA-treated samples of thirteen candidate reference genes obtained from published literature related to qRT-PCR expression profiling in soybean (Table 1, Dataset S1, Figure S1). These candidate genes were chosen based on their functional homology to genes that appear to demonstrate high expression stability in other plant systems $[44,45,54]$. Additionally, candidate genes were chosen which have been previously shown to demonstrate high stability in soybean under biotic stress conditions [46]. The $18 \mathrm{~S}$ ribosomal RNA gene was initially included among candidate reference genes for testing but was later excluded due to its extremely high abundance. Template cDNA for the samples analyzed with $18 S$ primers had to be diluted at least 1000 -fold relative to all other candidate genes to avoid the signal saturation obtained at low GT values. This dilution introduces a random element of variability which can potentially alter the apparent expression levels of target genes normalized with $18 \mathrm{~S}$.

To identify reference genes that can be widely used in gene expression analyses under various stresses, the data for all stress treatments were analyzed together for root and shoot tissues of young soybean seedlings by two methods: the delta CT and geNorm. These two types of tissues are preferably used for highthroughput gene expression profiling by qRT-PGR under abiotic stresses [39-41,55-58]. As shown in Table 2, data analysis using the delta CT method suggested that the five most stable genes in both root and shoot tissues under normal and stress conditions were $60_{s}>F b o x>E L F 1 b>A B C>I D E$. When the same datasets were analyzed using geNorm, a method used to determine gene expression stability (M) [59], four of the above five genes were also among the top five most stably expressed genes although the exact order was different $(60 s / A B C>I D E>$ Fbox $>E L F 1 a)$ (Figure 1A).

In real situation, we often study the differential expression of genes in a specific tissue rather than among various tissues. Thus, we subsequently looked for reference genes that performed well in individual tissues (roots or shoots) under various stresses. Analysis of the data using the delta CT method indicated that the most stably expressed genes in the root and shoots tissues are not the same, although they are overlapping (Table 2). For example, we found that ELF1b>60s $>A B C>F b o x>C Y P 2$ and $60 s>F b o x>E L F 1-$ $b>I D E>$ Act11 constituted the top five most stable genes in the roots and shoots, respectively. By geNorm method, the top five most stable genes for roots and shoots are $A B C / 60_{s}>E L F 1 b>>^{\prime \prime}$ CrP2 $>$ Fbox and Act11/Fbox $>I D E>A B C>60$ s, respectively (Figures $1 \mathrm{~B}$ and $1 \mathrm{C}$ ). The gene expression stability $(\mathbf{M})$ of the reference genes determined by geNorm was developed by Vandesompele et al. [59]. This method is based on the assumption that the expression ratio of two ideal reference genes remains constant in all samples and is unaffected by treatment conditions. Genes having the lowest $\mathrm{M}$ value are considered to be the most stable, while those with higher $\mathrm{M}$ value indicate less expression stability. The two methods of reference gene analysis have provided similar conclusions, thereby strengthening the legitimacy of the results obtained.

\section{Evaluation of reference gene stability in soybean root and shoot tissues under dehydration stress}

Since a reference gene which is the most stable in one stress may be highly variable under other stresses, thus we analyzed the data based on individual stresses to search for the most stable reference 

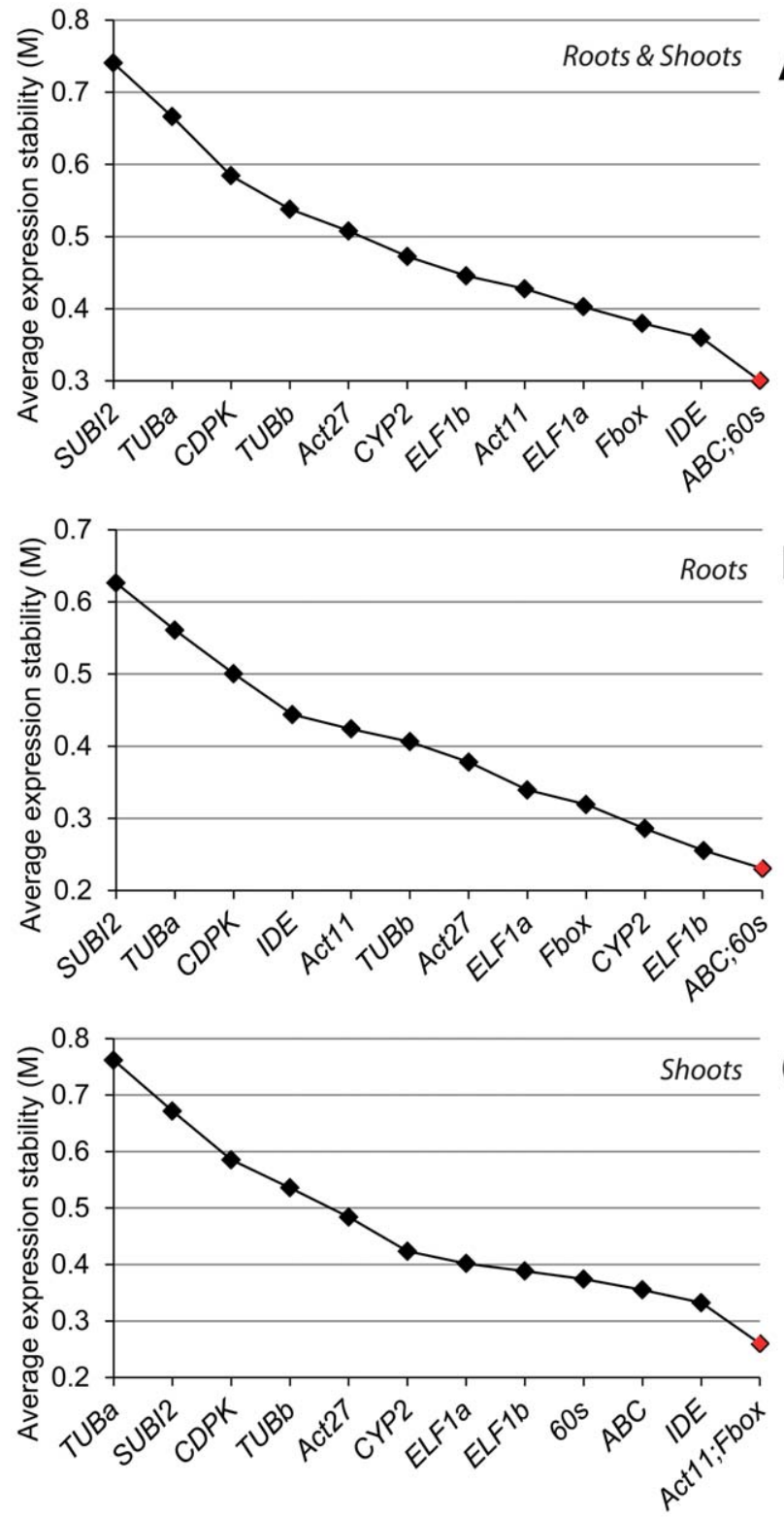

Figure 1. Expression stability of the candidate reference genes in root and shoot tissues of soybean seedlings under various abiotic stress and hormonal treatments. Soybean seedlings were subjected to dehydration, cold stress, salt stress and ABA treatments, and geNorm was used to assess expression stability in (A) treated roots and shoots; (B) treated roots; (C) treated shoots.

doi:10.1371/journal.pone.0046487.g001

gene(s) for each stress treatment. Under dehydration stress, results obtained from delta CT analysis (Table 3) revealed the top five most stably expressed genes for roots and shoots as $F b o x>$ $A B C>A c t 11>T U B b>A c t 27$ and Fbox $>60 s>E L F 1 b>A c t 11>I D E$, respectively. The top five genes that can perform best in both root and shoot tissues are Fbox $>60 s>I D E>A c t 11>A B C$. We then compared the data with that obtained by geNorm. In the roots, the gene encoding Fbox, ranked first by delta CT method, was not among the top five genes identified by geNorm which are Act11/ $A c t 27>A B C>T U B b>T U B a$ (Figure 2A). In the shoots, under dehydration stress Fbox was also ranked first by delta CT analysis; however, geNorm ranked $60 s / E L F 1 b$ as the best reference pair while Fbox was noted as the third best reference gene only
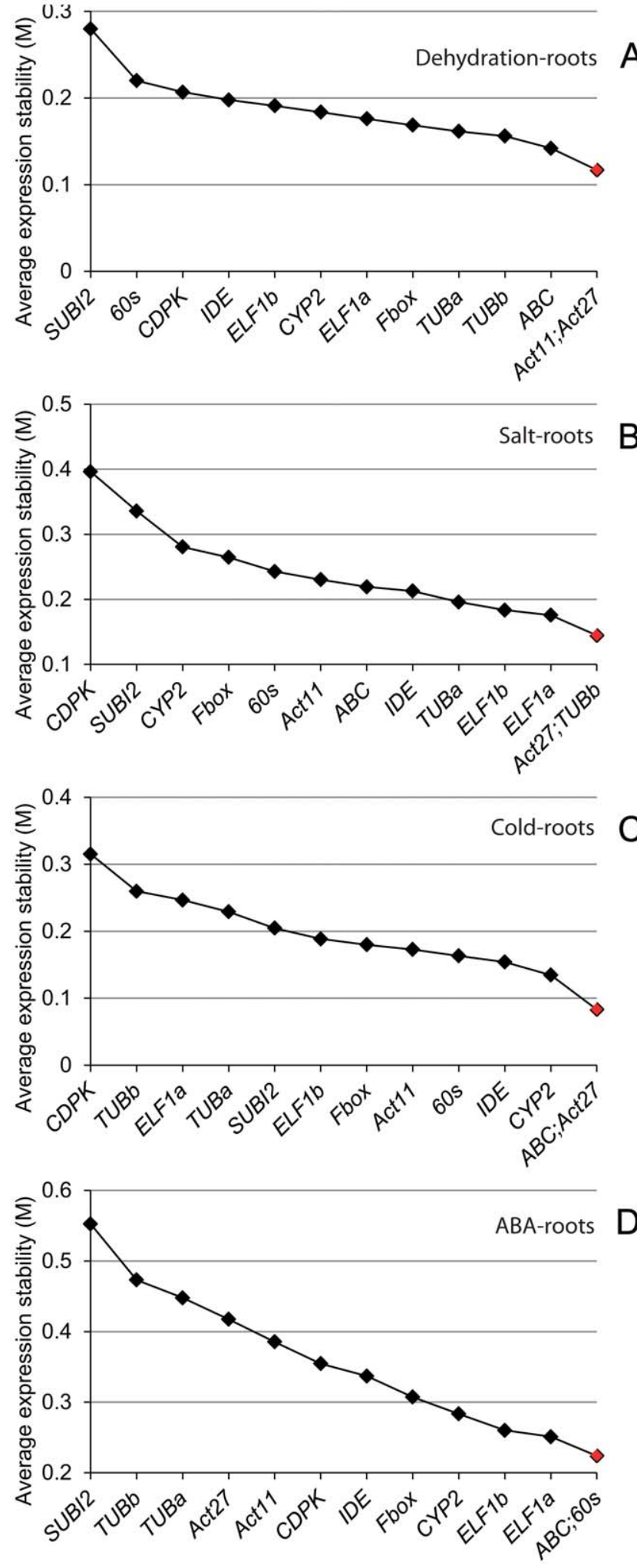

Figure 2. Expression stability and ranking for the candidate reference genes as determined by geNorm in the root tissues under individual stress or hormonal treatment. (A) Dehydration treatment. (B) Salt stress treatment. (C) Cold stress treatment. (D) ABA treatment.

doi:10.1371/journal.pone.0046487.g002 

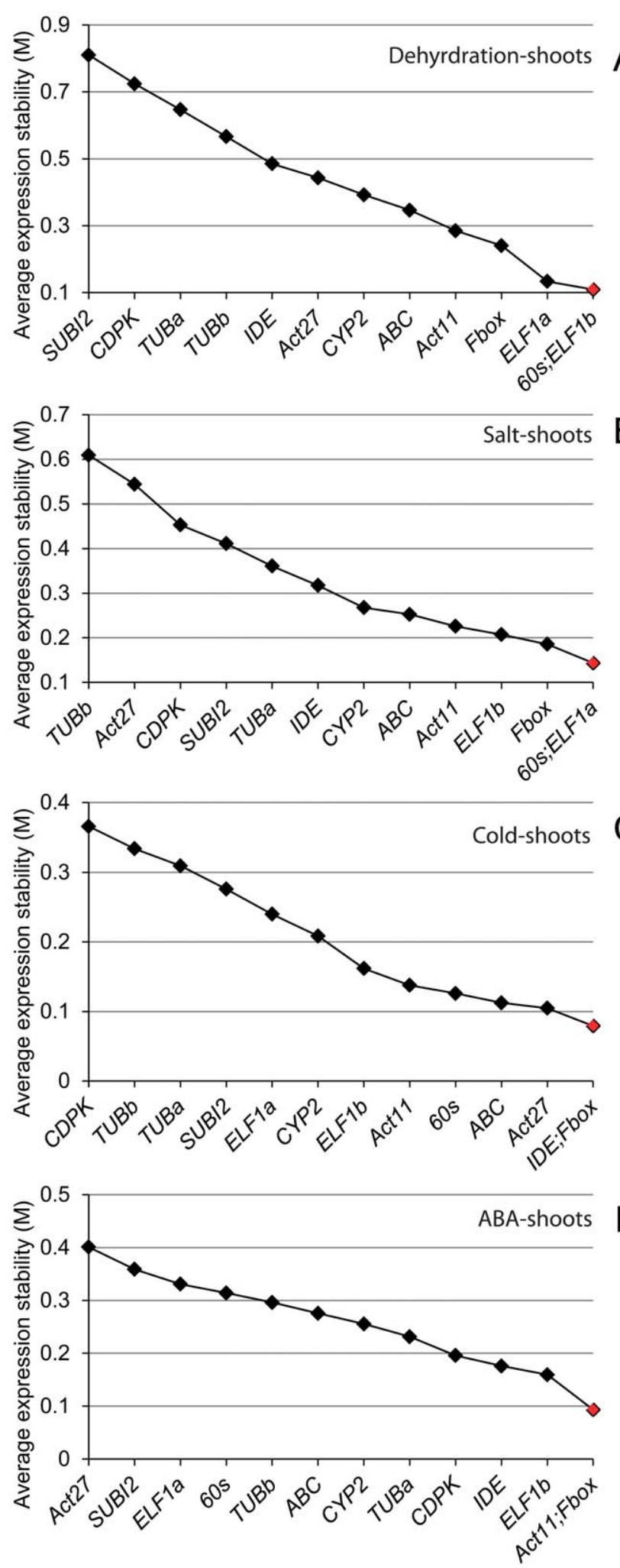

Figure 3. Expression stability and ranking for the candidate reference genes as determined by geNorm in the shoot tissues under individual stress or hormonal treatment. (A) Dehydration treatment. (B) Salt stress treatment. (C) Cold stress treatment. (D) ABA treatment.

doi:10.1371/journal.pone.0046487.g003
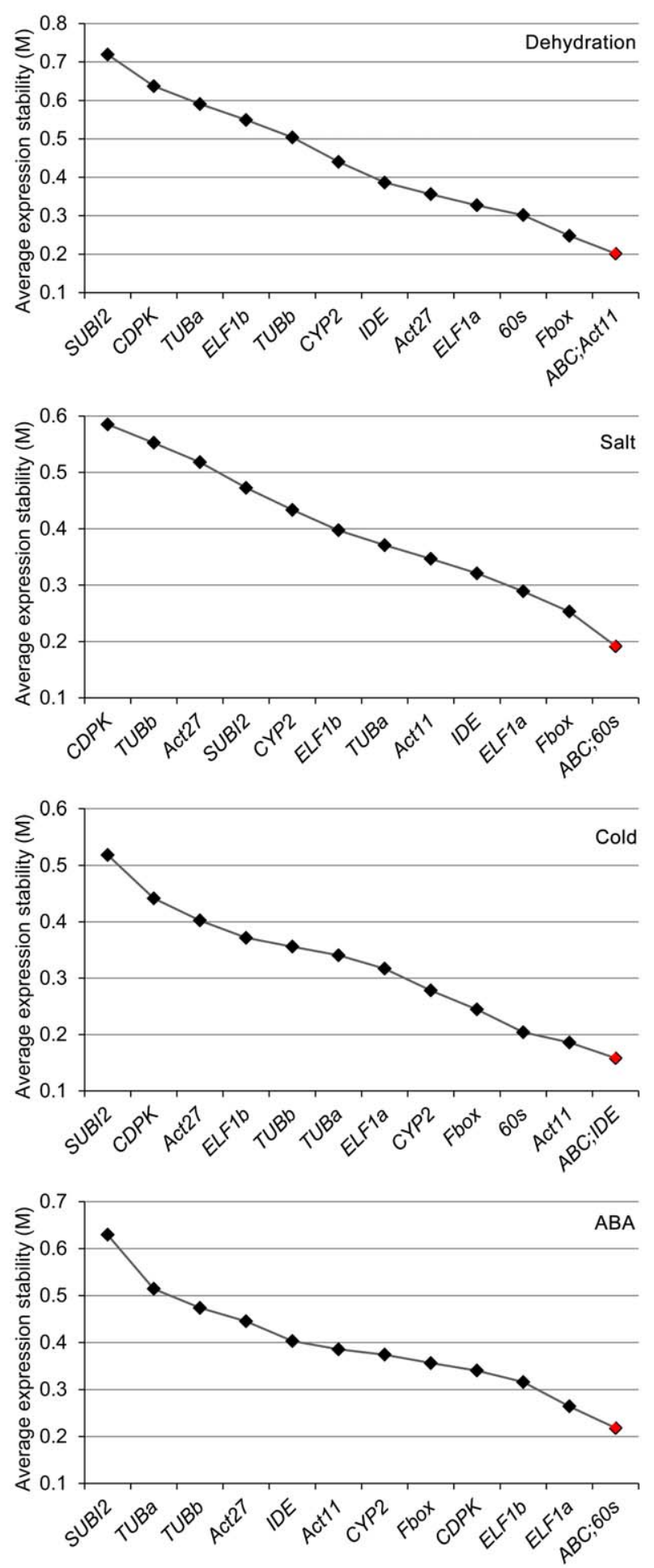

Figure 4. Expression stability and ranking for the candidate reference genes as determined by geNorm in both root and shoot tissues under individual stress or hormonal treatment. (A) Dehydration treatment. (B) Salt stress treatment. (C) Cold stress treatment. (D) ABA treatment.

doi:10.1371/journal.pone.0046487.g004

(Figure 3A). The top five genes that perform best in both dehydrated roots and shoots determined by geNorm are $A B C$ / Act11 $>$ Fbox $>60 s>$ ELF1a (Figure 4A). 
Table 3. Average of standard deviations of delta CT obtained from tissues under individual stress.

\begin{tabular}{|c|c|c|c|c|c|c|c|c|c|c|c|c|}
\hline & \multicolumn{3}{|c|}{ Dehydration } & \multicolumn{3}{|l|}{ Salt } & \multicolumn{3}{|l|}{ Cold } & \multicolumn{3}{|l|}{ ABA } \\
\hline & Roots & Shoots & $R \& S$ & Roots & Shoots & $R \& S$ & Roots & Shoots & $R \& S$ & Roots & Shoots & $R \& S$ \\
\hline $60 \mathrm{~s}$ & 0.2902 & 0.4857 & 0.5346 & 0.3015 & 0.3537 & 0.3579 & 0.2196 & 0.2579 & 0.2778 & 0.3653 & 0.3420 & 0.3996 \\
\hline$A B C$ & 0.2145 & 0.6269 & 0.6452 & 0.2896 & 0.4840 & 0.4180 & 0.2441 & 0.2647 & 0.3557 & 0.4050 & 0.3278 & 0.4318 \\
\hline Act27 & 0.2179 & 0.6418 & 0.7222 & 0.3128 & 0.7563 & 0.5739 & 0.2424 & 0.2564 & 0.4209 & 0.5245 & 0.5056 & 0.5560 \\
\hline Act11 & 0.2159 & 0.5537 & 0.6363 & 0.3823 & 0.4164 & 0.4384 & 0.2726 & 0.3457 & 0.3707 & 0.4838 & 0.3282 & 0.4563 \\
\hline CDPK & 0.2437 & 0.9915 & 0.7685 & 0.6666 & 0.7199 & 0.6857 & 0.5454 & 0.5116 & 0.5909 & 0.5288 & 0.3292 & 0.4780 \\
\hline CYP2 & 0.2807 & 0.6573 & 0.8326 & 0.3622 & 0.3792 & 0.5192 & 0.2449 & 0.3092 & 0.3632 & 0.4226 & 0.3485 & 0.4756 \\
\hline ELF1a & 0.2362 & 0.6186 & 0.8439 & 0.3238 & 0.4713 & 0.4462 & 0.2968 & 0.3152 & 0.4136 & 0.4192 & 0.3944 & 0.4537 \\
\hline ELF1b & 0.2378 & 0.5209 & 0.6804 & 0.2656 & 0.3277 & 0.4703 & 0.2448 & 0.2706 & 0.3563 & 0.3987 & 0.2959 & 0.4073 \\
\hline Fbox & 0.2026 & 0.4538 & 0.4943 & 0.3489 & 0.3268 & 0.3707 & 0.2404 & 0.2382 & 0.3120 & 0.4435 & 0.3221 & 0.4320 \\
\hline IDE & 0.2439 & 0.5967 & 0.5913 & 0.2743 & 0.4947 & 0.4441 & 0.2180 & 0.2991 & 0.3497 & 0.4787 & 0.3714 & 0.5126 \\
\hline$S U B / 2$ & 0.5441 & 1.2082 & 1.1174 & 0.5667 & 0.6558 & 0.6179 & 0.2898 & 0.3859 & 0.8414 & 0.9597 & 0.4756 & 1.1887 \\
\hline TUBa & 0.2268 & 1.0299 & 0.8549 & 0.2998 & 0.5392 & 0.4750 & 0.2779 & 0.3671 & 0.3670 & 0.5854 & 0.3893 & 0.6987 \\
\hline$T U B b$ & 0.2176 & 0.8387 & 0.7954 & 0.3212 & 0.7498 & 0.5985 & 0.2936 & 0.3724 & 0.3851 & 0.5330 & 0.3625 & 0.5416 \\
\hline
\end{tabular}

Data obtained for the top five genes are shown in bold letters, while those for the top two genes are in italic and bold letters.

doi:10.1371/journal.pone.0046487.t003

\section{Evaluation of reference gene stability in soybean root and shoot tissues under salt stress}

Unlike dehydration stress under which Fbox was identified as the best reference gene for roots, under salt stress Fbox was not among the top five genes for root tissues as determined by delta CT analysis (Table 3). Instead, ELF1b and $I D E$ were found to be the best reference gene pair among the top five genes $(E L F 1 b>I$ $D E>A B C>T U B a>60 s)$. In the salt stress-treated shoots, the top five genes are Fbox $>E L F 1 b>60 s>C Y P 2>A c t 11$. With the exception of the CIP2, the remaining genes were also ranked among top five genes in dehydration-treated shoots (Table 3). Although, ELF1b and Fbox were ranked first in the roots and shoots, respectively, when the data for roots and shoots were combined and analyzed, 60s was found to be the most stably expressed gene followed by Fbox (Table 3). When the data from the salt stresstreated roots were analyzed by geNorm, it turned out to be that the top three genes ranked by geNorm (Act27, TUBb and ELF1a) were not among the top five genes short-listed by delta CT analysis (Figure 2B, Table 3). In shoots, geNorm analysis indicated that except ELF1a, which was ranked first by geNorm but was out of the top five genes determined by delta CT analysis, the remaining top four genes, $60 s>F b o x>E L F 1 b>A c t 11$ were among the top five most stably expressed genes determined by delta CT analysis (Figure 3B, Table 3). The top two genes identified by delta CT method for both salt-treated roots and shoots (60s and Fbox, Table 3) were also among top three genes determined by geNorm (Figure 4B).

\section{Evaluation of reference gene stability in soybean root and shoot tissues under cold stress}

Soybean adaptive responses to cold stress have also attracted a great deal of attention $[51,60,61]$. Thus, we next searched for the best reference genes to be used for expression analysis under cold stress among the 13 selected candidate genes. Using delta CT analysis, the top five genes that are most stably expressed in the roots and shoots of soybean seedlings under cold stress were $I D E>60 s>F b o x>A c t 27>A B C$ and Fbox $>A c t 27>60 s>A B-$ $C>E L F 1 b$, respectively (Table 3 ). Four of the best performed reference genes ranked by delta CT analysis in each tissue were also shown to be the most stable genes by geNorm. Specifically, geNorm analysis demonstrated that $A B C /$ Act $27>C Y P 2>I D E>60$ s and $I D E / F b o x>A c t 27>A B C>60$ s are the top five best reference genes for roots and shoots, respectively, under cold stress (Figures 2G and 3C). Although the 60s gene was not the most stably expressed gene in any given tissues; however, it was the topranked gene to be used as a reference gene for cold stress across root and shoot tissues. The top five best candidate reference genes common for both tissues in cold stress were identified by delta CT analysis and geNorm are $60 s>F b o x>I D E>A B C>E L F 1 b$ (Table 3) and $A B C / I D E>A c t 11>60 s>$ Fbox (Figure 4G), respectively.

\section{Evaluation of reference gene stability in soybean root and shoot tissues under ABA treatment}

$\mathrm{ABA}$ has been established as a key hormone involved in the regulation of plant responses to various abiotic stresses, such as drought and high salinity. Under these stresses, endogenous ABA level is increased, leading to up-regulation of many stressresponsive genes in ABA-dependent manner [62]. Thus, ABA treatment is often included in expression studies together with other stress treatments to determine whether the change in gene expression in response to stresses is ABA-dependent or ABAindependent [39,51,52]. Hence, a good reference gene for expression studies of stress-responsive genes under abiotic stresses should also stably express under ABA treatment. This prompted us to rank the performance of the selected 13 candidate reference genes to obtain the most stably expressed genes in roots and shoots that had been subjected to exogenous ABA treatment. The five top-listed genes in the roots and shoots under ABA treatment as revealed by delta $\mathrm{CT}$ analysis were $60_{s}>E L F 1 b>A B C>E L F 1 a>$ $C Y P 2$ and $E L F 1 b>F b o x>A B C>A c t 11>C D P K$, respectively (Table 3). Only two genes, namely the $A B C$ and $E L F 1 b$, were overlapping in the lists of the top five most stably expressed genes in each tissue, suggesting that the most stable reference genes are not only stress/hormone-dependent but also tissue-dependent. When geNorm was use to analyze the expression stability, we found that all the top five genes listed for the roots $(A B C$ ) $60 s>E L F 1 a>E L F 1 b>C Y P 2)$ were also ranked as the top five by a 
Table 4. Differential expression of known dehydrationinducible genes normalized with 605 , Fbox or SUBI2 reference genes.

\begin{tabular}{|c|c|c|c|c|c|c|c|}
\hline & & \multicolumn{2}{|c|}{ GmNAC19 } & & & & \\
\hline & & \multicolumn{3}{|c|}{ Fold Changes } & \multicolumn{3}{|c|}{ Standard errors } \\
\hline & & SUBI2 & $60 \mathrm{~s}$ & Fbox & $S U B I 2$ & $60 s$ & Fbox \\
\hline $0 \mathrm{~h}$ & Root & 1.00 & 1.00 & 1.00 & 0.14 & 0.27 & 0.22 \\
\hline Dry $2 \mathrm{~h}$ & & 1.26 & 2.49 & 2.58 & 0.10 & 0.27 & 0.27 \\
\hline Dry $10 \mathrm{~h}$ & & 1.78 & 2.32 & 3.22 & 0.18 & 0.05 & 0.14 \\
\hline $0 \mathrm{~h}$ & Shoot & 1.00 & 1.00 & 1.00 & 0.11 & 0.04 & 0.04 \\
\hline Dry $2 \mathrm{~h}$ & & 0.96 & 3.13 & 3.75 & 0.15 & 0.09 & 0.06 \\
\hline \multirow[t]{2}{*}{ Dry $10 \mathrm{~h}$} & & 1.12 & 18.66 & 13.93 & 0.09 & 0.05 & 0.04 \\
\hline & & \multicolumn{3}{|c|}{ GmNAC43 } & & & \\
\hline $0 \mathrm{~h}$ & Root & 1.00 & 1.00 & 1.00 & 0.24 & 0.23 & 0.19 \\
\hline Dry $2 \mathrm{~h}$ & & 1.50 & 3.06 & 3.18 & 0.22 & 0.23 & 0.23 \\
\hline Dry $10 \mathrm{~h}$ & & 1.68 & 3.06 & 4.24 & 0.11 & 0.04 & 0.14 \\
\hline $0 \mathrm{~h}$ & Shoot & 1.00 & 1.00 & 1.00 & 0.04 & 0.05 & 0.05 \\
\hline Dry $2 \mathrm{~h}$ & & 17.26 & 25.42 & 30.51 & 0.16 & 0.12 & 0.08 \\
\hline \multirow[t]{2}{*}{ Dry $10 \mathrm{~h}$} & & 25.69 & 101.32 & 75.61 & 0.04 & 0.09 & 0.08 \\
\hline & & \multicolumn{2}{|c|}{ GmNAC85 } & & & & \\
\hline $0 \mathrm{~h}$ & Root & 1.00 & 1.00 & 1.00 & 0.24 & 0.23 & 0.18 \\
\hline Dry $2 \mathrm{~h}$ & & 3.06 & 6.23 & 6.46 & 0.16 & 0.18 & 0.16 \\
\hline Dry $10 \mathrm{~h}$ & & 4.96 & 9.02 & 12.50 & 0.24 & 0.15 & 0.24 \\
\hline $0 \mathrm{~h}$ & Shoot & 1.00 & 1.00 & 1.00 & 0.05 & 0.02 & 0.02 \\
\hline Dry $2 \mathrm{~h}$ & & 58.75 & 86.51 & 103.83 & 0.16 & 0.13 & 0.07 \\
\hline \multirow[t]{2}{*}{ Dry $10 \mathrm{~h}$} & & 139.24 & 549.09 & 409.76 & 0.02 & 0.08 & 0.06 \\
\hline & & \multicolumn{2}{|c|}{ GmNAC92 } & & & & \\
\hline $0 \mathrm{~h}$ & Root & 1.00 & 1.00 & 1.00 & 0.29 & 0.27 & 0.22 \\
\hline Dry $2 \mathrm{~h}$ & & 1.22 & 2.49 & 2.58 & 0.27 & 0.27 & 0.27 \\
\hline Dry $10 \mathrm{~h}$ & & 1.28 & 2.32 & 3.22 & 0.12 & 0.05 & 0.14 \\
\hline $0 \mathrm{~h}$ & Shoot & 1.00 & 1.00 & 1.00 & 0.02 & 0.04 & 0.04 \\
\hline Dry 2 h & & 2.12 & 3.13 & 3.75 & 0.13 & 0.09 & 0.06 \\
\hline Dry $10 \mathrm{~h}$ & & 4.73 & 18.66 & 13.93 & 0.02 & 0.05 & 0.04 \\
\hline
\end{tabular}

geNorm analysis although the order was different (Figure 2D, Table 3). As for the shoots, with the exception of the $A B C$, the other four top-listed genes determined by delta CT analysis were found among the top five genes as suggested by a geNorm analysis (Act11/Fbox $>$ ELF1b $>I D E>C D P K$ ) (Figure 3D, Table 3). In addition, we also searched for reference genes that can be used to compare gene expression across tissues under ABA treatment. The data for roots and shoots were combined and analyzed using the delta CT approach. Results on Table 3 demonstrated that the top five most stably expressed genes in both roots and shoots of soybean seedlings were $60 s>E L F 1 b>A B C>$ Fbox $>$ ELF1a. The same five genes were also identified by geNorm, although the exact order was different (Figure 4D).

\section{Validation of the usefulness of the reference genes identified from this study}

Next, to validate the performance of the reference genes identified in this study on known abiotic-stress inducible genes, we quantified the expression of four $G m N A C$ genes, which were reported to be up-regulated by dehydration [39,41], and normalized their expression levels using a representative least stable reference gene (SUBI2) and two representative good reference genes (Fbox and 60s) (Table 3). As shown in Table 4, with a fold-change threshold of 2.0 , we would have consistently failed to detect the induced expression in the roots and shoots for

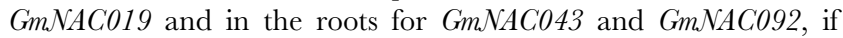
SUBI2 - a least stable reference gene under dehydration stress were used as a reference gene. However, the up-regulation of these genes was reliably detected when the expression levels were normalized with the most stable reference genes, such as Fbox or $60 s$. When the induction level was high, as that of GmNAC85 in both tissues or that of other $G m N A C$ genes in the shoots, normalizing to bad reference genes, such as SUBI2, could still be able to detect the induction but was shown to underestimate the induction level by 3- to 4-fold (Table 4).

\section{Conclusions}

Upon exposure to stresses, soybean plants activate numerous signaling pathways to respond to the adverse environmental stimuli [1-3]. The first step toward adapting to stresses is to induce or repress the expression of various genes. To accurately understand the mechanisms regulating stress responses at transcriptional level and to identify the appropriate stressresponsive genes, stably expressed reference genes are needed if qRT-PCR is used for expression profiling. Before this study was conducted, there have been no comprehensive reports on the search for reference genes for soybean under various abiotic stress conditions, except a study which surveyed for reference genes for expression study under drought stress in soybean. However, this study used only four candidate genes, namely the $G m \beta$-actin, GmGADPH, GmLectin and GmRNA18s, among which the Gm $\beta$-actin and GmRNA18s were found to be the best reference genes for soybean under their experimental conditions [47]. This prompted us to choose 13 candidate reference genes from literature and ranked their performance as the best reference genes under various abiotic stress and hormonal treatments, including dehydration, salt, cold and ABA treatments. Because most of the recent studies showed the preferable use of root and shoot tissues of soybean seedlings for gene expression profiling under abiotic stresses using qRT-PCR [39-41,55-58]; we tested the expression stability of the 13 selected candidate genes in these tissues of 12day old soybean plants. Our results revealed that there is no single reference gene that can be best for all conditions and/or both the tissues. Instead, we found that the best reference genes are tissueand/or stress-dependent. In addition, although there is high agreement in overall among the top five genes ranked by either delta CT analysis or geNorm, the best reference genes inferred by one method may not be exactly the same ones as determined by another approach; a phenomenon which has also been observed earlier $[63,64]$. As geNorm has been known to rank co-regulated genes as the best reference genes, our conclusions were made based primarily on the result of delta CT analysis. The result obtained by geNorm is provided to readers as additional information source. The most stably expressed reference genes identified in this study were shown to help detect subtle differential rates of gene expression as well as avoid the underestimation of the induction/repression levels.

Taken together, using delta CT analysis with consideration of geNorm result, we suggest that the following gene pairs (summarized in Table 5) are suitable for use as reference genes in the respective tissues and under specific stress: 
Table 5. Recommended reference genes for qRT-PCR of soybean tissues under various abiotic stresses.

\begin{tabular}{|c|c|c|c|c|c|c|}
\hline \multirow[b]{2}{*}{ Dehydration } & \multicolumn{2}{|l|}{ Roots } & \multicolumn{2}{|l|}{ Shoots } & \multicolumn{2}{|c|}{ Roots \& Shoots } \\
\hline & Fbox/ABC & ELF1b/60s & Fbox/60s & 60s/Fbox & Fbox/60s & 60s/Fbox \\
\hline Salt & $E L F 1 b / I D E$ & & Fbox/ELF1b & & 60s/Fbox & \\
\hline Cold & IDE/60s & & Fbox/Act27 & & 60s/Fbox & \\
\hline ABA & $60 \mathrm{~s} / \mathrm{ELF1b}$ & & ELF1b/Fbox & & $60 \mathrm{~s} / \mathrm{ELF} 1 \mathrm{~b}$ & \\
\hline
\end{tabular}

doi:10.1371/journal.pone.0046487.t005

(i) For dehydration, Fbox/ $A B C$ should be used for the roots and Fbox/60s for the shoots, respectively. The Fbox/60s gene pair is also the best for comparing expression between roots and shoots under normal and dehydration conditions.

(ii) For salt stress, ELF1b/IDE and Fbox/ELF1b should be used as reference genes for roots and shoots, respectively. For reference in both root and shoot tissues under salt stress, $60 s / F b o x$ is the best gene pair.

(iii) For studying expression change by cold stress individually in the roots or the shoots $I D E / 60$ s and Fbox/Act27 are the best reference pairs, respectively. For analysis gene expression under cold stress across root and shoot tissues, $60 s / F b o x$ should be used.

(iv) To examine gene expression under ABA stress in roots, shoots or across these two tissues, 60s/ELF1b, ELF1b/Fbox and 60 s/ELF1b are the best reference genes, respectively.

(v) Based on the results of this study, when comparison of the expression profiles of a gene in response to various stresses is of interest, the ELF1b/60s, 60s/Fbox and 60s/Fbox reference gene pairs are recommended for roots, shoots and both tissues, respectively. When a single reference gene is required, 60s should be the best choice.

\section{References}

1. Tran LS, Mochida K (2010) Functional genomics of soybean for improvement of productivity in adverse conditions. Funct Integr Genomics 10: 447-462.

2. Tran LS, Nguyen HT (2009) Future biotechnology of legumes. In: Emerich WD, Krishnan H, editors. Nitrogen fixation in crop production. Madison, WI: ASA-CSA-SSSA. pp. 265-308.

3. Thao NP, Tran LS (2011) Potentials toward genetic engineering of droughttolerant soybean. Crit Rev Biotechnol doi:10.3109/07388551.2011.643463.

4. Gutierrez-Gonzalez JJ, Guttikonda SK, Tran LS, Aldrich DL, Zhong R, et al. (2010) Differential expression of isoflavone biosynthetic genes in soybean during water deficits. Plant Cell Physiol 51: 936-948.

5. Neelakandan AK, Nguyen HT, Kumar R, Tran LS, Guttikonda SK, et al. (2010) Molecular characterization and functional analysis of Glycine max sterol methyl transferase 2 genes involved in plant membrane sterol biosynthesis. Plant Mol Biol 74: 503-518.

6. Hadiarto T, Tran LS (2011) Progress studies of drought-responsive genes in rice. Plant Cell Rep 30: 297-310.

7. Manavalan LP, Guttikonda SK, Tran LS, Nguyen HT (2009) Physiological and molecular approaches to improve drought resistance in soybean. Plant Cell Physiol 50: 1260-1276.

8. Tran LS, Nakashima K, Shinozaki K, Yamaguchi-Shinozaki K (2007) Plant gene networks in osmotic stress response: from genes to regulatory networks. Methods Enzymol 428: 109-128.

9. Nakashima K, Ito Y, Yamaguchi-Shinozaki K (2009) Transcriptional regulatory networks in response to abiotic stresses in Arabidopsis and grasses. Plant Physiol 149: 88-95.

10. Jogaiah S, Ramsandra Govind S, Tran LS (2012) System biology-based approaches towards understanding drought tolerance in food crops. Crit Rev Biotechnol doi: 10.3109/07388551.2012.659174.

11. Tran LS, Nishiyama R, Yamaguchi-Shinozaki K, Shinozaki K (2010) Potential utilization of NAC transcription factors to enhance abiotic stress tolerance in plants by biotechnological approach. GM Crops 1: 32-39.

12. Valliyodan B, Nguyen HT (2006) Understanding regulatory networks and engineering for enhanced drought tolerance in plants. Curr Opin Plant Biol 9: 189-195.

\section{Supporting Information}

Figure S1 Melting curves of the amplicons. The amplicons were produced by the primer pairs used to quantify the expression stability of the 13 candidate reference genes.

(TIF)

Table S1 Raw data of delta CT analysis of the expression stability of the candidate reference genes. (XLS)

Dataset S1 Primer locations on respective transcript. Primer sequences are in orange or in red boxes. Primer pairs with positions on two differently colored portions of the sequence are on two different exon or UTRs.

(PDF)

\section{Author Contributions}

Conceived and designed the experiments: DTL DLA L-SPT HTN. Performed the experiments: DTL YW CVH RN DLA BV SKG TNQ JJG-G L-SPT. Analyzed the data: DTL DLA L-SPT. Contributed reagents/materials/analysis tools: L-SPT HTN. Wrote the paper: DTL DLA BV L-SPT HTN. Revised the manuscript: DTL L-SPT.

13. Xin H, Qin F, Tran LS (2012) Transcription factors involved in environmental stress responses in plants. In: Ahmad P, Prasad MNV, editors. Environmental Adaptations and Stress Tolerance of Plants in the Era of Climate Change. New York: Springer. pp. 279-295.

14. Choudhary SP, Volkan Oral H, Bhardwaj R, Yu JQ, Tran LS (2012) Interaction of Brassinosteroids and Polyamines Enhances Copper Stress Tolerance in Raphanus Sativus. J Exp Bot doi: 10.1093/jxb/ers219.

15. Ma Y, Qin F, Tran LS (2012) Contribution of Genomics to Gene Discovery in Plant Abiotic Stress Responses. Molecular Plant doi: 10.1093/mp/sss085.

16. Choudhary SP, Yu JO Yamaguchi-Shinozaki K, Shinozaki K, Tran LS (2012) Benefits of brassinosteroid crosstalk. Trends Plant Sci. Available: http://dx.doi. org/10.1016/j.tplants.2012.05.012.

17. Ha S, Vankova R, Yamaguchi-Shinozaki K, Shinozaki K, Tran LS (2012 Cytokinins: metabolism and function in plant adaptation to environmental stresses. Trends Plant Sci 17: 172-179.

18. Le DT, Choi JD, Tran LS (2010) Amino acids conferring herbicide resistance in tobacco acetohydroxyacid synthase. GM Crops 1: 62-67.

19. Mochida K, Shinozaki K (2011) Advances in omics and bioinformatics tools for systems analyses of plant functions. Plant Cell Physiol 52: 2017-2038.

20. Alwine JC, Kemp DJ, Stark GR (1977) Method for detection of specific RNAs in agarose gels by transfer to diazobenzyloxymethyl-paper and hybridization with DNA probes. Proc Natl Acad Sci U S A 74: 5350-5354.

21. Tran LS, Nakashima K, Sakuma Y, Simpson SD, Fujita Y, et al. (2004) Isolation and functional analysis of Arabidopsis stress-inducible NAC transcription factors that bind to a drought-responsive cis-element in the early responsive to dehydration stress 1 promoter. Plant Cell 16: 2481-2498.

22. Tran LS, Urao T, Qin F, Maruyama K, Kakimoto T, et al. (2007) Functional analysis of AHK1/ATHK 1 and cytokinin receptor histidine kinases in response to abscisic acid, drought, and salt stress in Arabidopsis. Proc Natl Acad Sci U S A 104: 20623-20628.

23. Stolf-Moreira R, Lemos EGM, Carareto-Alves L, Marcondes J, Pereira SS, et al. (2011) Transcriptional Profiles of Roots of Different Soybean Genotypes Subjected to Drought Stress. Plant Molecular Biology Reporter 29: 19-34. 
24. Seki M, Narusaka M, Ishida J, Nanjo T, Fujita M, et al. (2002) Monitoring the expression profiles of 7000 Arabidopsis genes under drought, cold and highsalinity stresses using a full-length cDNA microarray. Plant J 31: 279-292.

25. Kreps JA, Wu Y, Chang HS, Zhu T, Wang X, et al. (2002) Transcriptome changes for Arabidopsis in response to salt, osmotic, and cold stress. Plant Physiol 130: 2129-2141.

26. Tran LS, Nakashima K, Sakuma Y, Osakabe Y, Qin F, et al. (2007) Coexpression of the stress-inducible zinc finger homeodomain ZFHD1 and NAC transcription factors enhances expression of the ERD1 gene in Arabidopsis. Plant J 49: 46-63.

27. Nishiyama R, Le DT, Watanabe Y, Matsui A, Tanaka M, et al. (2012) Transcriptome analyses of a salt-tolerant cytokinin-deficient mutant reveal differential regulation of salt stress response by cytokinin deficiency. PLoS One 7: e32124.

28. Qin F, Kodaira KS, Maruyama K, Mizoi J, Tran LS, et al. (2011) SPINDLY, a negative regulator of gibberellic acid signaling, is involved in the plant abiotic stress response. Plant Physiol 157: 1900-1913.

29. Kodaira KS, Qin F, Tran LS, Maruyama K, Kidokoro S, et al. (2011) Arabidopsis Cys2/His2 zinc-finger proteins AZF1 and AZF2 negatively regulate abscisic acid-repressive and auxin-inducible genes under abiotic stress conditions. Plant Physiol 157: 742-756.

30. Ballman KV (2008) Genetics and genomics: gene expression microarrays. Circulation 118: 1593-1597.

31. Tang T, Francois N, Glatigny A, Agier N, Mucchielli MH, et al. (2007) Expression ratio evaluation in two-colour microarray experiments is significantly improved by correcting image misalignment. Bioinformatics 23: 2686-2691.

32. To TK, Nakaminami K, Kim JM, Morosawa T, Ishida J, et al. (2011) Arabidopsis HDA6 is required for freezing tolerance. Biochemical and Biophysical Research Communications 406: 414-419.

33. Matsui A, Ishida J, Morosawa T, Mochizuki Y, Kaminuma E, et al. (2008) Arabidopsis transcriptome analysis under drought, cold, high-salinity and ABA treatment conditions using a tiling array. Plant Cell Physiol 49: 1135-1149.

34. Matsui A, Ishida J, Morosawa T, Okamoto M, Kim JM, et al. (2010) Arabidopsis tiling array analysis to identify the stress-responsive genes. Methods Mol Biol 639: 141-155.

35. Czechowski T, Bari RP, Stitt M, Scheible WR, Udvardi MK (2004) Real-time RT-PCR profiling of over 1400 Arabidopsis transcription factors: unprecedented sensitivity reveals novel root- and shoot-specific genes. Plant Journal 38: 366379 .

36. Nolan T, Hands RE, Bustin SA (2006) Quantification of mRNA using real-time RT-PCR. Nature Protocols 1: 1559-1582.

37. Ginzinger DG (2002) Gene quantification using real-time quantitative PCR: An emerging technology hits the mainstream. Experimental Hematology 30: 503512.

38. Udvardi MK, Czechowski T, Scheible WR (2008) Eleven golden rules of quantitative RT-PCR. Plant Cell 20: 1736-1737.

39. Tran LS, Quach TN, Guttikonda SK, Aldrich DL, Kumar R, et al. (2009) Molecular characterization of stress-inducible GmNAC genes in soybean. Mol Genet Genomics 281: 647-664.

40. Le DT, Nishiyama R, Watanabe Y, Mochida K, Yamaguchi-Shinozaki K, et al. (2011) Genome-wide expression profiling of soybean two-component system genes in soybean root and shoot tissues under dehydration stress. DNA Res 18: 17-29.

41. Le DT, Nishiyama R, Watanabe Y, Mochida K, Yamaguchi-Shinozaki K, et al. (2011) Genome-wide survey and expression analysis of the plant-specific NAC transcription factor family in soybean during development and dehydration stress. DNA Res 18: 263-276.

42. Pereira SS, Guimaraes FC, Carvalho JF, Stolf-Moreira R, Oliveira MC, et al. (2011) Transcription factors expressed in soybean roots under drought stress. Genet Mol Res 10: doi: 10.4238/2011.October.4221.4235.

43. Gutierrez L, Mauriat M, Guenin S, Pelloux J, Lefebvre JF, et al. (2008) The lack of a systematic validation of reference genes: a serious pitfall undervalued in reverse transcription-polymerase chain reaction (RT-PCR) analysis in plants. Plant Biotechnol J 6: 609-618.

44. Jian B, Liu B, Bi Y, Hou W, Wu C, et al. (2008) Validation of internal control for gene expression study in soybean by quantitative real-time PCR. BMC Mol Biol 9: 59 .
45. Hu R, Fan C, Li H, Zhang Q, Fu YF (2009) Evaluation of putative reference genes for gene expression normalization in soybean by quantitative real-time RT-PCR. BMC Mol Biol 10: 93.

46. Libault M, Thibivilliers S, Bilgin DD, Radwan O, Benitez M, et al. (2008) Identification of four soybean reference genes for gene expression normalization. The Plant Genome 1: 44-54.

47. Stolf-Moreira R, Lemos EGM, Abdelnoor RV, Beneventi MA, Rolla AAP, et al. (2011) Identification of reference genes for expression analysis by real-time quantitative PCR in drought-stressed soybean. Pesquisa Agropecuária Brasileira 46: 58-65.

48. Ramakers C, Ruijter JM, Deprez RH, Moorman AF (2003) Assumption-free analysis of quantitative real-time polymerase chain reaction (PCR) data. Neurosci Lett 339: 62-66.

49. Ruijter JM, Ramakers C, Hoogaars WM, Karlen Y, Bakker O, et al. (2009) Amplification efficiency: linking baseline and bias in the analysis of quantitative PCR data. Nucleic Acids Res 37: e45.

50. Silver N, Best S, Jiang J, Thein SL (2006) Selection of housekeeping genes for gene expression studies in human reticulocytes using real-time PCR. BMC Mol Biol 7: 33.

51. Gao SQ, Chen M, Xu ZS, Zhao CP, Li L, et al. (2011) The soybean GmbZIP1 transcription factor enhances multiple abiotic stress tolerances in transgenic plants. Plant Mol Biol 75: 537-553.

52. Nishiyama R, Watanabe Y, Fujita Y, Le DT, Kojima M, et al. (2011) Analysis of cytokinin mutants and regulation of cytokinin metabolic genes reveals important regulatory roles of cytokinins in drought, salt and abscisic acid responses, and abscisic acid biosynthesis. Plant Cell 23: 2169-2183.

53. Aslam M, Grover A, Sinha VB, Fakher B, Pande V, et al. (2012) Isolation and characterization of cold responsive NAC gene from Lepidium latifolium. Mol Biol Rep DOI: $10.1007 / \mathrm{s} 1$ 1033-012-1828-0.

54. Irsigler AS, Costa MD, Zhang P, Reis PA, Dewey RE, et al. (2007) Expression profiling on soybean leaves reveals integration of ER- and osmotic-stress pathways. BMC Genomics 8: 431.

55. Stolf-Moreira R, Medri ME, Neumaier N, Lemos NG, Brogin RL, et al. (2010) Cloning and quantitative expression analysis of drought-induced genes in soybean. Genet Mol Res 9: 858-867.

56. Nouri MZ, Hiraga S, Yanagawa Y, Sunohara Y, Matsumoto H, et al. (2012) Characterization of calnexin in soybean roots and hypocotyls under osmotic stress. Phytochemistry 74: 20-29.

57. Xie ZM, Zou HF, Lei G, Wei W, Zhou QY, et al. (2009) Soybean Trihelix transcription factors GmGT-2A and GmGT-2B improve plant tolerance to abiotic stresses in transgenic Arabidopsis. PLoS One 4: e6898.

58. Wei W, Huang J, Hao YJ, Zou HF, Wang HW, et al. (2009) Soybean GmPHDtype transcription regulators improve stress tolerance in transgenic Arabidopsis plants. PLoS One 4: e7209.

59. Vandesompele J, De Preter K, Pattyn F, Poppe B, Van Roy N, et al. (2002) Accurate normalization of real-time quantitative RT-PCR data by geometric averaging of multiple internal control genes. Genome Biol 3: RESEARCH0034.

60. Toda K, Takahashi R, Iwashina T, Hajika M (2011) Difference in chillinginduced flavonoid profiles, antioxidant activity and chilling tolerance between soybean near-isogenic lines for the pubescence color gene. J Plant Res 124: 173182.

61. Maruyama K, Todaka D, Mizoi J, Yoshida T, Kidokoro S, et al. (2012) Identification of cis-acting promoter elements in cold- and dehydration-induced transcriptional pathways in Arabidopsis, rice, and soybean. DNA Res 19: 37-49.

62. Fujita Y, Fujita M, Shinozaki K, Yamaguchi-Shinozaki K (2011) ABA-mediated transcriptional regulation in response to osmotic stress in plants. J Plant Res 124: 509-525.

63. Kortner TM, Valen EC, Kortner H, Marjara IS, Krogdahl A, et al. (2011) Candidate reference genes for quantitative real-time PCR (qPCR) assays during development of a diet-related enteropathy in Atlantic salmon (Salmo salar L.) and the potential pitfalls of uncritical use of normalization software tools. Aquaculture 318: 355-363.

64. Mehdi Khanlou K, Van Bockstaele E (2012) A critique of widely used normalization software tools and an alternative method to identify reliable reference genes in red clover (Trifolium pratense L.). Planta doi: 10.1007/ s00425-012-1682-2. 